\title{
Minimum Redundancy MIMO Radars
}

\author{
Chun-Yang Chen and P. P. Vaidyanathan \\ Dept. of Electrical Engineering, MC 136-93 \\ California Institute of Technology, Pasadena, CA 91125, USA \\ E-mail: cyc@caltech.edu,ppvnath@systems.caltech.edu
}

\begin{abstract}
The multiple-input multiple-output (MIMO) radar concept has drawn considerable attention recently. In the traditional single-input multiple-output (SIMO) radar system, the transmitter emits scaled versions of a single waveform. However, in the MIMO radar system, the transmitter transmits independent waveforms. It has been shown that the MIMO radar can be used to improve system performance. Most of the MIMO radar research so far has focused on the uniform array. However, it is in general a loss of optimality to assume the array to be uniform. In this paper, the nonuniform array design problem in the MIMO radar is studied. In the SIMO radar, it has been shown that there is a class of linear arrays which minimizes the number of redundant spacings in the array. These are called minimum redundancy linear arrays. It has been shown that this class of arrays has excellent performance in rejection of mainlobe interferences. In this paper, the idea of minimum redundancy linear array is extended to the MIMO radar case. The numerical examples show that the proposed minimum redundancy MIMO radar results in improved rejection of mainlobe interferences, with negligible degradation in sidelobe interference rejection capabilities.
\end{abstract}

Index Terms - MIMO Radar, Nonlinear Arrays, Minimum Redundancy Linear Arrays, Adaptive Beamforming.

\section{INTRODUCTION}

Multiple-input multiple-output (MIMO) radars have drawn considerable attention recently for a number of their advantages, including high spatial resolution [7], excellent interference rejection capability [10], improved parameter identifiability [11], and enhanced flexibility for transmit beampattern design [12]. In the traditional single-input multiple-output (SIMO) radar system, the transmitter emits scaled versions of a single waveform. In the MIMO radar, the transmitter transmits independent waveforms in each of the transmitting antenna elements. It has been shown that the ability of transmitting independent waveforms can be used to enhance various system performances. One of the advantages is that the MIMO radar can be used to form a virtual array to greatly improve the spatial resolution [7]. It can be done by transmitting orthogonal waveforms and extracting the waveforms in each receiving element by using a set of matched filters. It has been shown that the matched filter output is equivalent to the signals received by an array with $N M$ elements, where $N$ is the number of the receiving antenna elements and $M$ is the number of the transmitting antenna elements. This array is called the virtual array.

It is known that nonuniform spacing may lead to significantly improved performance [9]. There are many approaches to design the nonlinear array [9]. One such approach is based

\footnotetext{
${ }^{1}$ Work supported in parts by the ONR grant N00014-06-1-0011, and the California Institute of Technology.
}

on the redundancy concept [1]. One can view an array as a grating or compound interferometer. The array can be utilized by considering all possible pairings of elements, with each pair acting as an interferometer. Given $N$ elements, there are $N(N-1) / 2$ possible pairings but not all of these are distinct. The minimum redundancy (MR) array uses the minimum number of antenna elements to generate a certain set of parings (as elaborated in Sec III), by minimizing duplicate pairs. By using this idea, one can construct a large aperture array with very few elements. Because of its excellent spatial resolution, it can be applied to detect fast moving targets in a SAR system [6]. The performance of the adaptive beamforming of the MR array has been examined in [5]. It has been shown that the MR array has excellent rejection of mainlobe interference, with negligible degradation in sidelobe interference rejection capabilities.

The purpose of this paper is to extend the MR idea to the case of MIMO radars. A MR MIMO radar design problem will be introduced. For small aperture, this problem can be solved by an exhaustive search algorithm. An example of MR MIMO radar will be presented and its performance will be examined. The rest of this paper is organized as follows. Section II reviews the idea of the virtual array. Section III extends the MR idea to the case of MIMO radars. Section IV examines the SINR performance of adaptive beamforming in a MR MIMO radar system and compares this to a uniform MIMO radar system. Finally, Section V concludes the paper.

Notations. Matrices are denoted by capital letters in boldface (e.g., A). Vectors are denoted by lowercase letters in boldface (e.g., x). Superscript ${ }^{\dagger}$ denotes transpose conjugation.

\section{Review of the Virtual ARray Concept}

One of the main advantages of MIMO radar is that the degrees of freedom can be greatly increased by the concept of virtual array [7]. Consider a linear transmitting array with $M$ antenna elements and a linear receiving array with $N$ antenna elements. The transmitting and the receiving arrays are parallel and co-located. The $m$ th transmitting antenna is located at $x_{T, m}$ and the $n$th receiving antenna is located at $x_{R, n}$. Fig. 1 (a) and (b) show an example with $M=3$ and $N=4$. The $m$ th transmitting antenna emits the waveform $\phi_{m}(t)$. The emitted waveforms are orthogonal, that is,

$$
\int \phi_{m}(\tau) \phi_{k}^{*}(\tau) d \tau=\delta_{m k}
$$

In each receiving antenna, these orthogonal waveforms are extracted by $M$ matched filters. Therefore, the total number of extracted signal equals $N M$. Consider a far-field point target. 
(a)<smiles>C1CC2CC1C2</smiles>

\section{$\bigvee_{0}$}

(b)

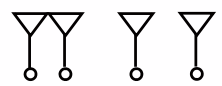

(c)
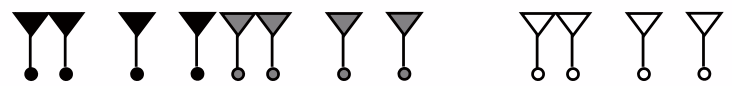

Fig. 1. Illustration of a MIMO radar system: (a) Transmitting array (b) Receiver array, and (c) Virtual array, with $M=3$ and $N=4$.

The target response in the $m$ th matched filter output of the $n$th receiving antenna can be expressed as

$$
s_{n, m}=\exp \left(j \frac{2 \pi}{\lambda} \sin \theta\left(x_{T, m}+x_{T, n}\right)\right),
$$

where $\theta$ is the looking direction of the target from the radar station. We have normalized the amplitude of the signal reflected by the target to unity. One can see that the phase differences are created by both the transmitting antenna locations and the receiving antenna locations. The target response in (1) is the same as the target response received by a receiving array with $N M$ antenna elements located at

$$
\begin{array}{r}
\left\{x_{T, m}+x_{R, n} \mid n=0,1, \cdots, N-1,\right. \\
m=0,1, \cdots, M-1\} .
\end{array}
$$

This $N M$-element array is called a virtual array. Fig 1 (c) shows the corresponding virtual array of the MIMO radar system illustrated in (a) and (b). Thus, we can create an $N M$ element virtual array by using only $N+M$ physical antenna elements. This will greatly increase the spatial resolution of the system.

\section{Minimum Redundancy MiMO RAdAR}

The minimum redundancy (MR) linear array was first proposed by Moffet [1]. The idea is to minimize the number of antenna elements by reducing the redundancy of the spacings. In this section, we extend the MR idea to the case of MIMO radar. Before further discussing the MR MIMO radar, we first show an example of the uniform MIMO radar. By choosing $x_{R, n}=\frac{\lambda}{2} n$ and $x_{T, m}=\frac{\lambda}{2} N m$, the virtual array in (2) becomes

$$
\left\{x_{T, m}+x_{R, n}\right\}=\left\{0, \frac{\lambda}{2}, \frac{\lambda}{2} \cdot 2, \cdots, \frac{\lambda}{2}(N M-1)\right\}
$$

This is a uniform array with spacing $\frac{\lambda}{2}$. The spacing is chosen to be $\frac{\lambda}{2}$ to avoid spatial aliasing. Fig. 2 shows an example of a uniform MIMO radar system with $M=3$ and $N=5$. In the uniform MIMO radar case, the received target signal in (1) becomes

$$
s_{k}=\exp (j \pi \sin \theta k)=e^{j \omega k}
$$

where we use $k$ to denote the index of the virtual array and $\omega \triangleq \pi \sin \theta$. For a beamformer $\mathbf{w}$, the beampattern can be
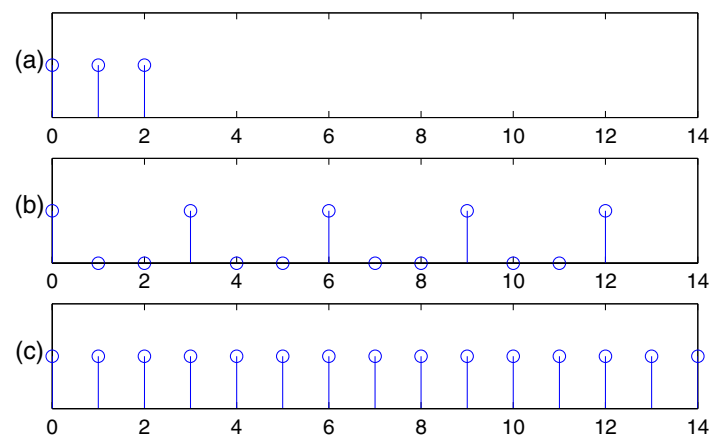

Fig. 2. Illustration of virtual array in a uniform MIMO radar: (a) transmitting array, (b) receiving array, and (c) virtual array.

expressed as

$$
B(\omega) \triangleq\left|\mathbf{w}^{\dagger} \mathbf{s}\right|=\left|\sum_{k=0}^{N M-1} w_{k} e^{j \omega k}\right|,
$$

One can see that the beampattern is the frequency magnitude response of the weighting coefficients $w_{k}$. Therefore, the beampattern design problem is equivalent to the FIR filter design problem when the antenna array is chosen to be a uniform array. For a more general case, let

$$
x_{T, m}=u_{T, m} \frac{\lambda}{2}, \text { and } x_{R, n}=u_{R, n} \frac{\lambda}{2},
$$

where $u_{T, m}$ and $u_{R, n}$ are some integers. In this case, the beampattern becomes

$$
B(w)=\left|\sum_{k=0}^{N M-1} w_{k} e^{j \omega u_{k}}\right|,
$$

where

$$
\begin{aligned}
\left\{u_{k}\right\}= & \left\{u_{R, m}+u_{T, n} \mid\right. \\
& n=0,1, \cdots, N, m=0,1, \cdots, M\} .
\end{aligned}
$$

Equation (4) can be viewed as the magnitude response of another FIR filter $\mathbf{w}^{\prime}$, where

$$
w_{n}^{\prime}= \begin{cases}w_{k}, & n=u_{k} \text { for some } k \\ 0, & \text { otherwise. }\end{cases}
$$

The filter $\mathbf{w}^{\prime}$ can be viewed as a longer FIR filter with some coefficients restricted to be zero. It is well-known that a longer filter can produce shaper transition band. Since some of the coefficients will be restricted to zero, this will also affect the beampattern.

In [1], the concept of minimum redundancy (MR) array was introduced. It suggests that one should minimize the number of the array elements as long as the spacings between pairs of array elements includes all the integers between 1 and $L$, where $L$ is the desired normalized aperture and the spacings are defined as $\left\{u_{k}-u_{k^{\prime}}\right\}$, where $\left\{u_{k}\right\}$ are the normalized 
antenna locations. In the SIMO radar case, the MR array can be found by the following optimization problem:

$$
\begin{aligned}
& \min _{\left\{u_{k}\right\}} N \\
& \text { subject to }\left|\left\{u_{k}\right\}\right|=N \\
& \qquad\left\{u_{k}-u_{k^{\prime}}\right\} \supset\{1,2, \cdots, L\},
\end{aligned}
$$

where $|\mathcal{A}|$ denotes the cardinality of the set $\mathcal{A}$. This problem is a combinatorial problem which is in general not easy to solve. This problem is also of some interest in the theory of numbers, and it has been examined in [3]. Fig. 3 (a) shows an example of a MR array with five elements and a normalized aperture equal to nine. Fig. 3 (b) shows the histogram of its spacing (between pairs of elements). One can see that each spacing is measured by at least one pair of array elements. In

(a)

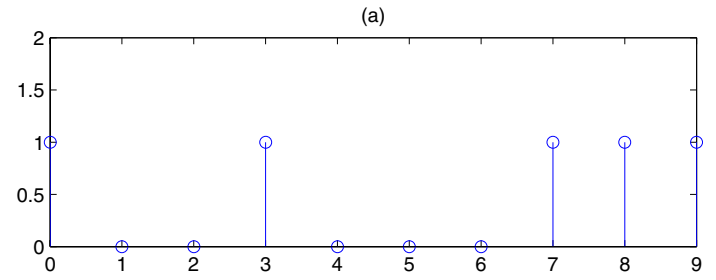

(b)

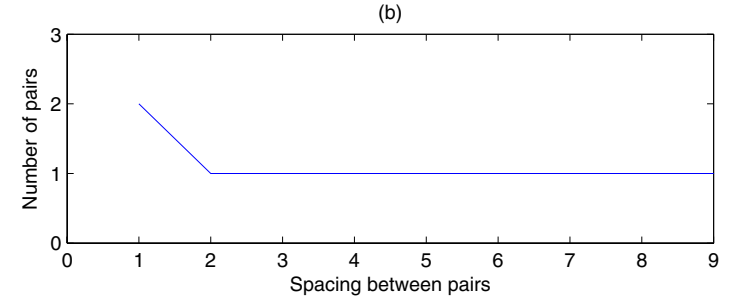

Fig. 3. Illustration of a minimum redundancy array: (a) array locations, (b) histogram of the spacing.

the MIMO radar case, by using (5), the spacings $\left\{u_{k}-u_{k^{\prime}}\right\}$ can be expressed as

$$
\begin{aligned}
& \left\{u_{T, m}-u_{R, n}-u_{T, m^{\prime}}+u_{R, n^{\prime}} \mid\right. \\
& \left.m, m^{\prime}=0,1, \cdots, M, n, n^{\prime}=0,1, \cdots, N\right\}
\end{aligned}
$$

Therefore, for a given desired normalized aperture $L$, the transmitting and the receiving array of the MR MIMO radar can be found by solving the following optimization problem:

$$
\begin{aligned}
& \quad \min _{\left\{u_{T, m}\right\},\left\{u_{R, n}\right\}} N+M \\
& \text { subject to }\left|\left\{u_{T, m}\right\}\right|=M,\left|\left\{u_{R, m}\right\}\right|=N, \\
& \quad\left\{u_{T, m}-u_{R, n}-u_{T, m^{\prime}}+u_{R, n^{\prime}}\right\} \supset\{1,2, \cdots, L\} .
\end{aligned}
$$

This problem is more complex than the SIMO version in (6). For a smaller $L$, one can use an exhaustive search algorithm to find the solution. Fig. 4 shows an example of a solution with $L=63$. The solution is $\left\{u_{T, m}\right\}=\{0,1,3\}$ and $\left\{u_{R, n}\right\}=\{0,6,13,40,60\}$. It is obtained by an exhaustive search algorithm. To simplify the search algorithm, we restrict

$$
u_{T, m}-u_{R, n}-u_{T, m^{\prime}}+u_{R, n^{\prime}} \leq L \quad \forall m, m^{\prime}, n, n^{\prime} .
$$
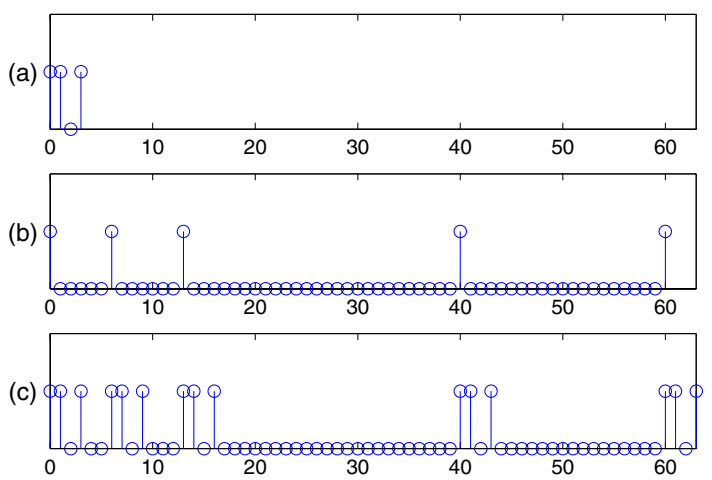

Fig. 4. Illustration of virtual array in a minimum redundancy MIMO radar: (a) transmitting array, (b) receiving array, and (c) virtual array.

This is similar to what is proposed in [4] for traditional radar. Note that with this restriction, the solution is only suboptimal. one can relax this restriction and search the solution on a grid with large enough number of points. However, the exhaustive search may take an extremely long time.

Comparing Fig. 2 and 4, one can see that the MR virtual array has a much longer aperture while the number of antenna elements in both systems are the same. It requires a MIMO radar system with at least sixteen antenna elements (with eight in the transmitter and eight in the receiver) to form a uniform virtual array with a normalized aperture $L=63$. However, the MR MIMO radar in this example requires only a total of eight antenna elements. Fig. 5 shows the histograms of the spacings of the MR and the uniform MIMO radar systems. One can see
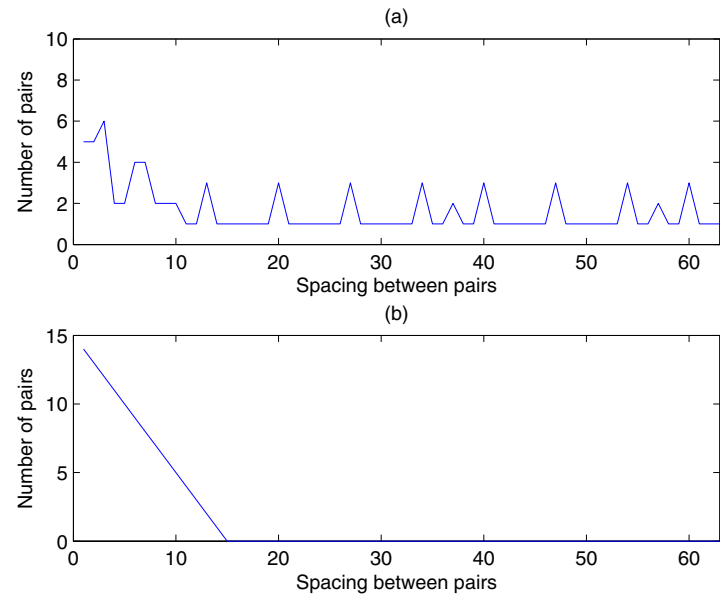

Fig. 5. Histogram of the spacings for (a) minimum redundancy MIMO radar in Fig. 4, and (b) uniform MIMO radar in Fig. 2.

that the histogram for the MR MIMO radar system is flatter and covers all the integers from 1 to $\mathrm{L}$. 


\section{Simulations}

In this section, we compare the SINR performance of the $\mathrm{MR}$ and the uniform MIMO radar systems. The arrays in Fig. 2 and 4 are compared. Consider a desired far-field point target at angle $\theta=0^{\circ}$ and three undesired far-field point objects at angles $\left[2^{\circ}, 15^{\circ},-60^{\circ}\right]$. Note that the angle of the first object is very close to the angle of the target. We normalize the variance of the thermal noise to $0 \mathrm{~dB}$. The target signal power is also $0 \mathrm{~dB}$ and the power of the signals reflected by the other three point objects are $[10,10,20]$ dB. Fig. 6 shows the beampattern of the minimum variance distortionless (MVDR) beamformers [2] of both the MR and uniform MIMO radar systems. The MVDR beamformer is obtained by solving the

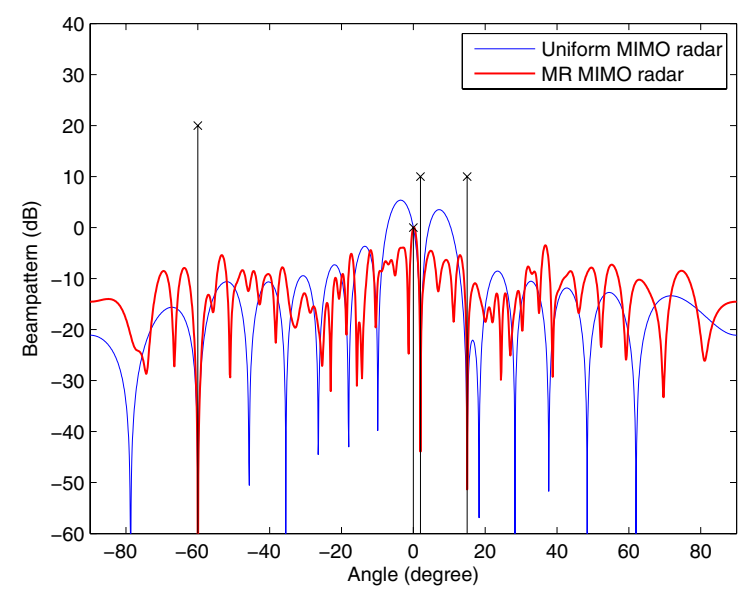

Fig. 6. Comparison of beampatterns for the case with mainlobe interference.

following optimization problem [2]

$$
\begin{aligned}
& \min _{\mathbf{w}} \mathbf{w}^{\dagger} \mathbf{R} \mathbf{w} \\
& \text { subject to } \mathbf{w}^{\dagger} \mathbf{s}=1,
\end{aligned}
$$

where $\mathbf{s}$ is a vector which contains the target response in (1) and $\mathbf{R}$ is the covariance matrix of the received signals. The SINR is defined as

$$
\rho=\frac{\left|\mathbf{w}^{\dagger} \mathbf{s}\right|^{2} \sigma_{s}^{2}}{\mathbf{w}^{\dagger} \mathbf{R} \mathbf{w}-\left|\mathbf{w}^{\dagger} \mathbf{s}\right|^{2} \sigma_{s}^{2}},
$$

where $\sigma_{s}^{2}$ is the target signal power. In this example, the SINR of the uniform MIMO system is $4.70 \mathrm{~dB}$ while the SINR of the MR MIMO radar system is $9.74 \mathrm{~dB}$. One can see that the MR MIMO system has a much sharper mainlobe. Therefore it can effectively reject the undesired object at the angle of $2^{\circ}$. This is why its SINR performance is more than $5 \mathrm{~dB}$ better than the uniform MIMO system. Fig. 7 shows another example where we move the first object from $2^{\circ}$ to $-20^{\circ}$. In this case, the SINR of the uniform MIMO radar system is $11.70 \mathrm{~dB}$ and the SINR of the MR MIMO radar system is $11.19 \mathrm{~dB}$. It appears that the uniform MIMO radar system has better sidelobe rejection ability. However, the difference of the SINR performance in this case is negligible.

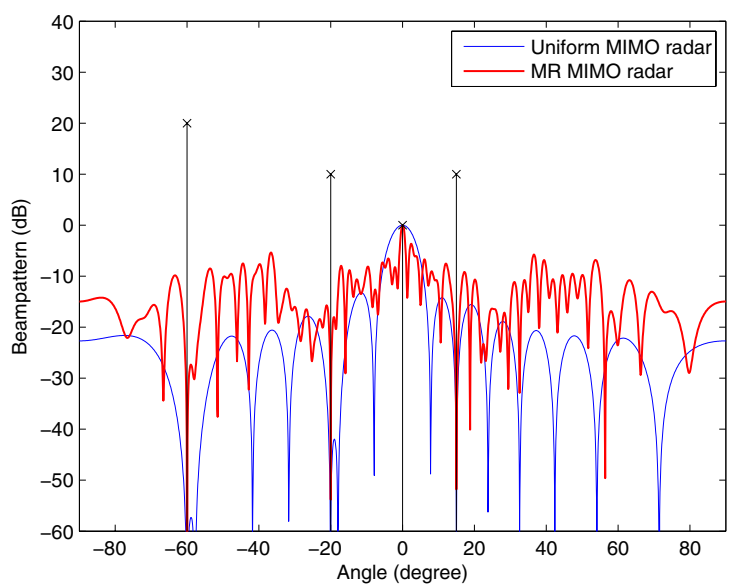

Fig. 7. Comparison of beampatterns for the case without mainlobe interference.

\section{CONCLUSIONS}

In this paper, we have extended the idea of minimum redundancy $(\mathrm{MR})$ arrays to the case of the MIMO radar. By reducing multiple occurrence of identical spacings, one can minimize the number of the antenna elements while obtaining the same array aperture. The MR MIMO radar array geometry can be found by exhaustive search. Numerical examples show that the proposed minimum redundancy MIMO radar results in improved rejection of mainlobe interferences, with negligible degradation in sidelobe interference rejection capabilities.

\section{REFERENCES}

[1] A. T. Moffet, "Minimum-Redundancy Linear Arrays," IEEE Trans. on Antennas and Propagation, Volume 16, Issue 2, Mar 1968.

[2] J. Capon, "High-resolution frequency-wavenumber spectrum analysis," Proc. IEEE, vol. 57, no. 8, pp. 1408-1418, Aug. 1969.

[3] J. Leech, "On the representation of $1,2, \cdots, n$ by differences," J. Landon Math. Soc., 31:160-169, April 1956.

[4] K. A. Blanton and J. H. McClellan "New search algorithm for minimum redundancy linear arrays," IEEE International Conference on Acoustics, Speech, and Signal Processing, vol. 2, p.p. 1361-1364, 14-17 April 1991.

[5] M. B. Jorgenson, M. Fattouche, and S. T. Nichols, "Applications of Minimum Redundancy Arrays in Adaptive Beamforming," IEE Proceedings on Microwaves, Antennas and Propagation Volume 138, Issue 5, pp 441447, Oct. 1991

[6] Y. Zuo, G. Li, J. Xu, Y. -N. Peng, and X. -G. Xia, "Detection and Location of Fast Moving Targets Using Minimum Redundancy Linear Array SAR,' International Conference on Radar, pp 1-4, Oct. 2006.

[7] D. W. Bliss and K. W. Forsythe, "Multiple-Input Multiple-Output (MIMO) Radar and Imaging: Degrees of Freedom and Resolution," Proc. 37th IEEE Asilomar Conf. on Signals, Systems, and Computers, vol. 1, pp. 54-59, Nov. 2003.

[8] K. W. Forsythe, D. W. Bliss, and G. S. Fawcett, "Multiple-Input MultipleOutput (MIMO) radar: Performance Issues," Proc. 38th IEEE Asilomar Conf. on Signals, Systems, and Computers, vol. 1, pp. 7-10, Nov. 2004.

[9] S. Haykin, J. P. Reilly, V. Kezys, and E. Vertatschitsch, "Some aspects of array signal processing," IEE Proceedings on Radar and Signal Processing, Volume 139, Issue 1, pp. 1-26, Feb. 1992.

[10] Chun-Yang Chen and P. P. Vaidyanathan, "MIMO Radar Space-Time Adaptive Processing Using Prolate Spheroidal Wave Functions," IEEE Trans. on Signal Processing, to appear.

[11] J. Li, P. Stoica, L. Xu, and W. Roberts, "On Parameter Identifiability of MIMO Radar," IEEE Signal Processing Letters, to appear.

[12] P. Stoica, J. Li, and Y. Xie, "On Probing Signal Design For MIMO Radar," IEEE Trans. on Signal Processing Volume 55, Issue 8, Aug. 2007. 